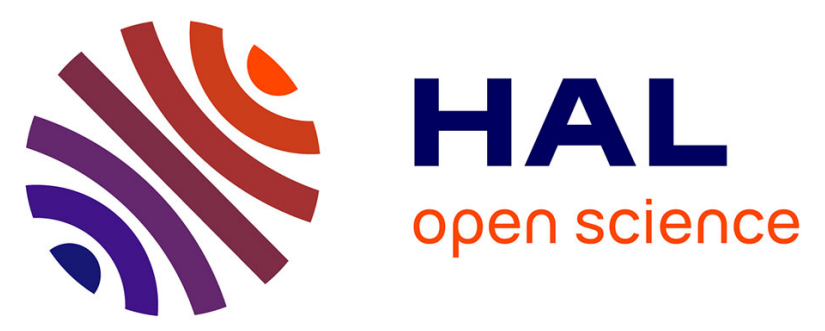

\title{
Highly Transparent and Conductive Indium-Free Vanadates Crystallized at Reduced Temperature on Glass Using a 2D Transparent Nanosheet Seed Layer
}

Alexis Boileau, Simon Hurand, F. Baudouin, U. Luders, Marie Dallocchio, Bruno Bérini, Aimane Cheikh, Adrian David, Fabien Paumier, Thierry

Girardeau, et al.

\section{To cite this version:}

Alexis Boileau, Simon Hurand, F. Baudouin, U. Luders, Marie Dallocchio, et al.. Highly Transparent and Conductive Indium-Free Vanadates Crystallized at Reduced Temperature on Glass Using a 2D Transparent Nanosheet Seed Layer. Advanced Functional Materials, 2022, 32 (5), pp.2108047. 10.1002/adfm.202108047 . hal-03340142

\section{HAL Id: hal-03340142 \\ https://hal.science/hal-03340142}

Submitted on 16 Sep 2021

HAL is a multi-disciplinary open access archive for the deposit and dissemination of scientific research documents, whether they are published or not. The documents may come from teaching and research institutions in France or abroad, or from public or private research centers.
L'archive ouverte pluridisciplinaire HAL, est destinée au dépôt et à la diffusion de documents scientifiques de niveau recherche, publiés ou non, émanant des établissements d'enseignement et de recherche français ou étrangers, des laboratoires publics ou privés.

$$
\text { Copyright }
$$


See discussions, stats, and author profiles for this publication at: https://www.researchgate.net/publication/354484653

\section{Highly Transparent and Conductive Indium-Free Vanadates Crystallized at Reduced Temperature on Glass Using a 2D Transparent Nanosheet Seed Layer}

Article in Advanced Functional Materials · September 2021

DOl: 10.1002/adfm.202108047

\section{CITATIONS}

20 authors, including:

Alexis Boileau

National Graduate School of Engineering and Research Center (Caen)

22 PUBLICATIONS 102 CITATIONS

SEE PROFILE

Florent Baudouin

Université de Rennes 1

7 PUBLICATIONS 9 CITATIONS

SEE PROFILE
READS

38

Simon Hurand

Université de Poitiers

37 PUBLICATIONS 699 CITATIONS

SEE PROFILE

Marie Dallocchio

Centre National De Recherche En Sciences Des Matériaux

5 PUBLICATIONS 3 CITATIONS

SEE PROFILE

Some of the authors of this publication are also working on these related projects:

Project ANR Polynash View project

Project Growth of rare earth vanadates REVO3 elaborated by magnetron sputtering and properties View project 


\title{
Highly Transparent and Conductive Indium-Free Vanadates Crystallized at Reduced Temperature on Glass Using a 2D Transparent Nanosheet Seed Layer
}

\author{
Alexis Boileau, Simon Hurand, Florent Baudouin, Ulrike Lüders, Marie Dallocchio, \\ Bruno Bérini, Aimane Cheikh, Adrian David, Fabien Paumier, Thierry Girardeau, \\ Philippe Marie, Christophe Labbé, Julien Cardin, Damien Aureau, Mathieu Frégnaux, \\ Maryline Guilloux-Viry, Wilfrid Prellier, Yves Dumont, Valérie Demange, and Arnaud Fouchet**
}

Indium-tin-oxide (ITO) is a widely employed transparent conducting oxide (TCO), but the indium scarcity and price encourage developing some alternatives. The correlated metals $\mathrm{CaVO}_{3}$ and $\mathrm{SrVO}_{3}$ have been recently identified as new TCOs with functional properties being comparable to ITO. However, their technological potential is limited by the critical requirement of a perovskite structure of the film, impossible to achieve via direct growth on substrates commonly used for applications. In this article, the authors tackle this limitation by demonstrating the crystalline growth of vanadate TCOs on glass at temperatures below $600{ }^{\circ} \mathrm{C}$, with the help of 2D nanosheets as transparent seed layers. The functional properties do not suffer from the textured structure of the films, as confirmed by an in-depth spectroscopic ellipsometry study, allowing for an industrially viable approach to integrate vanadate TCOs on virtually any surface and to exploit their promising performances as a new generation TCO.

\section{Introduction}

Transparent conducting oxides (TCOs) are an important class of materials for a wide range of technological applications such as flat screens or haptic technologies, solar cells, light

A. Boileau, U. Lüders, M. Dallocchio, A. Cheikh, A. David,

W. Prellier, A. Fouchet

Normandie Université

Ensicaen

UNICAEN

CNRS

CRISMAT

Caen 14000 , France

E-mail: arnaud.fouchet@ensicaen.fr

S. Hurand, F. Paumier, T. Girardeau

Institut Pprime

UPR 3346 CNRS-Université de Poitiers-ENSMA

SP2MI, Futuroscope-Chasseneuil, Cedex 86962, France

F. Baudouin, M. Guilloux-Viry, V. Demange

Université de Rennes

CNRS

ISCR - UMR 6226, ScanMAT - UMS 2001, Rennes F-35000, France

The ORCID identification number(s) for the author(s) of this article can be found under https://doi.org/10.1002/adfm.202108047. emitting diodes, electrochromic cells, or transparent antennas. ${ }^{[1,2]}$ The reference material, indium-tin-oxide (ITO), shows excellent optical transparencies reaching $80 \%$ and electrical resistivities below $1 \times 10^{-4} \Omega \mathrm{cm} \cdot{ }^{[3-5]}$ However, indium is a byproduct of other metals, far from abundant, showing strong price variations, and may result in supply difficulties despite high global criticality. ${ }^{[6-8]}$ Therefore, the research for indium-free TCOs has been an active field for some decades now. ${ }^{[9-13]}$ Recently, new indium-free TCOs were demonstrated, based on vanadate perovskites. ${ }^{[14]} \mathrm{CaVO}_{3}$ (CVO) and $\mathrm{SrVO}_{3}$ (SVO) are metallic systems with strong electronic correlations, leading to an enhanced effective mass, which shifts the plasma frequency out of the visible range. ${ }^{[14-16]}$ The functional properties of epitaxial thin films were shown to be comparable to those of ITO, positioning vanadates as a serious alternative for ITO. ${ }^{[14,17-22]}$ The composition of vanadates is made of elements easily available on earth, dividing the cost by 6 , based on the stock price of In $(157.5 \$ / \mathrm{kg})$ and V $(26.2 \$ / \mathrm{kg})$

DOI: $10.1002 / \mathrm{adfm} .202108047$ 
in June 2020. Hence, the vanadates compete directly with silver nanowires (AgNWs) films, identified for some years now as performing transparent conducting materials that can be deposited on any substrate at low temperature. ${ }^{[23]}$

Nevertheless, the technological potential of the vanadate TCOs is limited by the mandatory crystalline form to exhibit electrical conduction. ${ }^{18]}$ The direct growth on $\mathrm{Si}$ has been reported, but is hard to reproduce, ${ }^{[24,25]}$ due to chemical destabilization of SVO at the interface with Si leading to the formation of impurities. ${ }^{[26-29]}$ The use of a buffer layer could therefore be an alternative but the crystallization temperatures are typically rather high, ${ }^{[24,25]}$ jeopardizing the integrity of the possible device. In this article, we demonstrate the possibility to grow crystalline TCO films at low temperature on any interesting surface for optical applications and study the functional properties of such films.

\section{Results and Discussion}

Our approach is the use of nanosheet (NS) growth templates. ${ }^{[30]}$ Due to their thickness of only few nanometers, NS are optically transparent, being therefore compatible with transparent electronics. NS are obtained by an exfoliation process of various layered oxides ${ }^{[31]}$ and transferred by Langmuir-Blodgett (LB) method (Figure 1a,b ${ }^{[32]}$ on substrates that, when used bare, are non-adapted for complex oxide deposition, such as silicon or glass. The exceptionally rich structural diversity of NS makes them interesting growth templates for oxides ${ }^{[30,33-40]}$ or metals. ${ }^{[41]}$ SVO has a cubic perovskite structure with a lattice parameter $a=0.384(1) \mathrm{nm}^{[42]}$ whereas CVO is orthorhombically (o) distorted with pseudo-cubic (pc) $a_{\mathrm{pc}}=a_{\mathrm{o}} / \sqrt{ } 2=b_{\mathrm{o}} / \sqrt{ } 2=c_{\mathrm{o}} / 2 \approx 0.377(1) \mathrm{nm} \cdot{ }^{[43]}$ An adapted NS system would therefore be $\mathrm{Ca}_{2} \mathrm{Nb}_{3} \mathrm{O}_{10}{ }^{-}$(CNO) niobate nanosheets with an in-plane square lattice and a parameter $a_{\mathrm{NS}}=0.3854 \mathrm{~nm}^{[40]}$ (Figure 1a), leading to a misfit of $-0.34 \%$ for SVO and $-2.14 \%$ for CVO. Moreover, CNO NS exhibit a good thermal stability, of up to $800{ }^{\circ} \mathrm{C},{ }^{[31,35]}$ suitable for the growth of epitaxial $\mathrm{CVO}$ and SVO films crystallizing at temperatures of $\approx 400{ }^{\circ} \mathrm{C}$. ${ }^{[18]}$

The sketch drawn in Figure 1c gives a schematic illustration of the stacking model developed in this work to stabilize the vanadate perovskite structures on glass following the experimental procedure described in the Experimental section. The CNO NS seed layer of a few nanometers was deposited on Eagle XG Corning glass substrate (GS) (commonly used for flat panel displays ${ }^{[44,45]}$ ) by LB dip coating, followed by a CVO or SVO layer $(40 \mathrm{~nm})$ deposited by pulsed laser deposition (PLD) between 400 and $700{ }^{\circ} \mathrm{C}$ and an amorphous $\mathrm{LaAlO}_{3}$ (a-LAO) capping layer $(5 \mathrm{~nm})$ in order to avoid the spontaneous oxidation of the vanadate under the surrounding medium.

Atomic force microscopy (AFM) characterizations displayed in Figure 1d and scanning electron microscopy (SEM) for larger scales (see Supporting Information) provide a clear picture of the morphology. After deposition of the complete stack, the root mean square roughness of the films is very low, that is, below $1 \mathrm{~nm}$ apart from the films deposited at $700{ }^{\circ} \mathrm{C}$ (see Table S1, Supporting Information). Regarding the NS layer deposited by LB, the NS lateral size is between $100 \mathrm{~nm}$ to $2 \mu \mathrm{m}$ for the largest ones. The exploded view in Figure 1e highlights the stacking of three single NS layers with a decreasing coverage
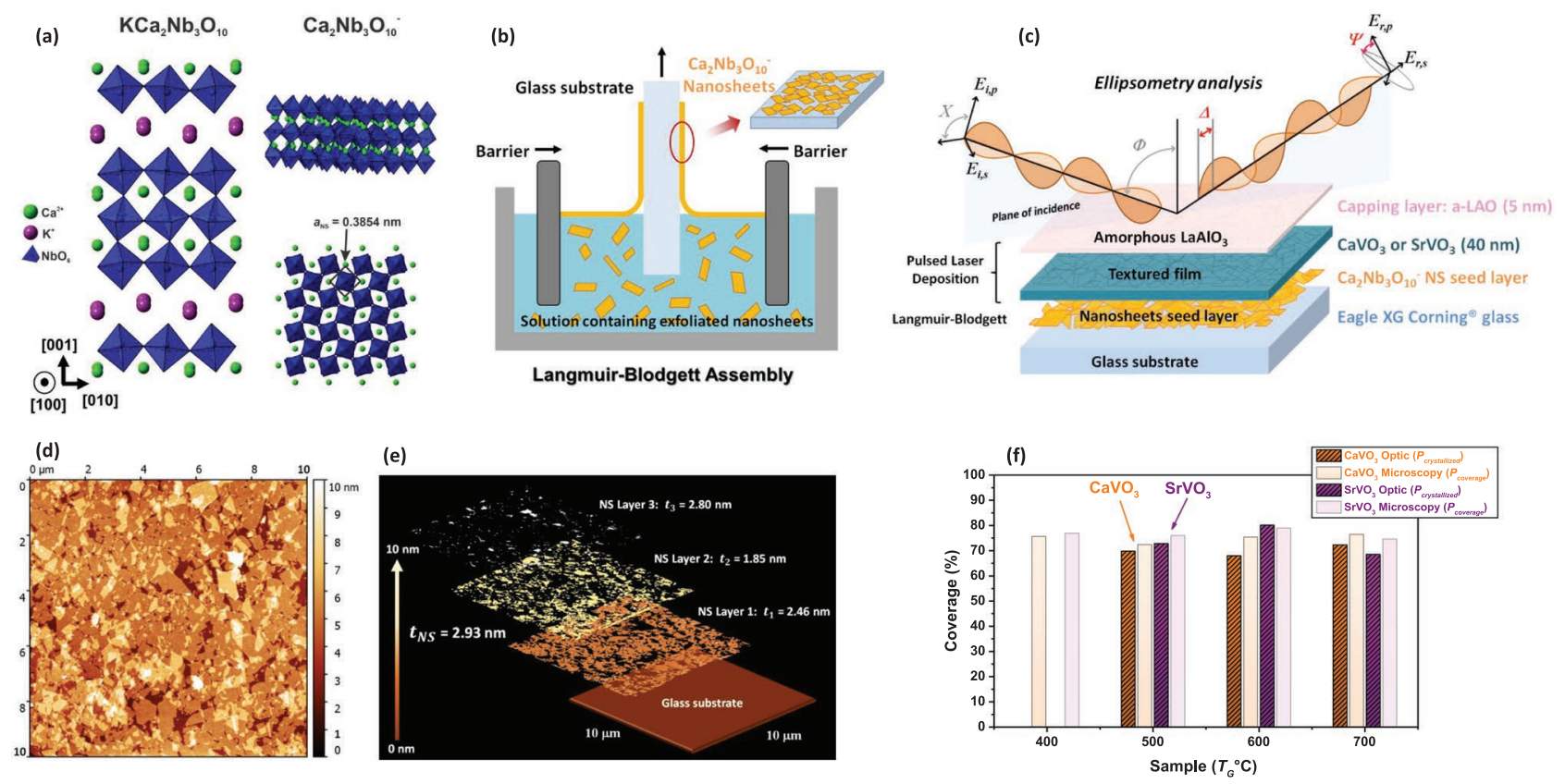

Figure 1. Preparation of the NS seed layer and synthesis of vanadate thin films. a) Crystalline representation of the parent material $\mathrm{KCa}_{2} \mathrm{Nb}_{3} \mathrm{O}_{10}$ and the delaminated CNO NS structure. b) Scheme of the LB method used to deposit CNO NS on glass. c) Sketch of the stacking model of the vanadate perovskites on NS-covered glass substrates and scheme of the associated SE analysis. d) $10 \times 10 \mu \mathrm{m}^{2}$ atomic force microscopy image of NS seed layer deposited on glass and e) exploded view showing the three extracted NS layers. The average thickness of the NS seed layer is $2.93 \mathrm{~nm}$. $\mathrm{f}$ ) Representation of the surface coverage by NS determined by microscopy techniques (coupled SEM-AFM analyses) and SE modeling as a function of the growth temperature $T_{\mathrm{G}}$. 
from the bottom upwards. This can be explained by the LB process, repeated several times to obtain a satisfying coverage, leading to the superposition of NS in some parts of the substrate. The average thickness of the entire NS seed layer $t_{\mathrm{NS}}$ is $2.93 \mathrm{~nm}$ (see details in Supporting Information). By comparison, spectroscopic ellipsometry (SE) measurements analyzed with an appropriate optical model (see details in Supporting Information) indicate an average thickness of the NS seed layer of $3.3 \mathrm{~nm}$, which is in perfect agreement. Therefore, the NS layer is constituted by an average of two single superposed CNO sheets with an individual thickness of $\approx 1.6 \mathrm{~nm}$.

As the vanadate films crystallize only on the substrate areas covered by the NS, and not directly on the glass surface (even at $700{ }^{\circ} \mathrm{C}$, see Supporting Information), an important parameter acting on the functional properties is the surface coverage $\left(P_{\text {coverage }}\right)$ by the NS. This parameter can be extracted from the SEM and AFM images (Supporting Information) leading to typical values of $P_{\text {coverage }} \approx 74 \%$ (Figure 1e). Besides, with an effective medium model (anisotropic Bruggeman effective medium approximation, ABEMA) in SE analyses, the proportion of amorphous vanadate phase $\left(P_{\text {amorphous }}\right)$ can be extracted (Supporting Information). The percentage of the crystallized phase in the layer $P_{\text {crystallized }}=100-P_{\text {amorphous }}$ are similar for all samples $(\approx 70 \%)$, in good accordance with $P_{\text {coverage }}$ (see Figure 1f).

We show in Figure $2 \mathrm{a}$ and $2 \mathrm{~b}$ the X-ray diffraction (XRD) patterns of CVO and SVO films. The crystallization temperature on NS occurs between 400 and $500{ }^{\circ} \mathrm{C}$ (see Figure 2a,b), which is in the same range as SVO films on mono-crystalline substrates. ${ }^{[18,19]}$ Low growth temperature $T_{\mathrm{G}}$ is helpful to prevent the substrate from thermal damages, but also to avoid structural modifications of the NS. Indeed, a high diffracting intensity is measured at $500{ }^{\circ} \mathrm{C}$ indicating an excellent structural crystallinity of the film, decreasing continuously up to $700{ }^{\circ} \mathrm{C}$. This deterioration may be explained by a structural degradation of the CNO NS with $T_{\mathrm{G}}{ }^{\left[{ }^{[31]}\right.}$ but also by the glass transformation above the strain point $\left(669^{\circ} \mathrm{C}\right)$, which cannot be completely ruled out for thin films deposited at $700{ }^{\circ} \mathrm{C}$. In particular, at $T_{\mathrm{G}}$ higher than $650{ }^{\circ} \mathrm{C}$, we evidenced by energy dispersive X-ray spectroscopy (EDXS) coupled to transmission electron microscopy (TEM) and depth chemical profiling by X-ray photoelectron spectroscopy (XPS) (see Supporting Information) the cationic out-diffusion of $\mathrm{Si}$ and $\mathrm{Nb}$ into the vanadate films, a mechanism well identified leading to the degradation of the perovskite structure and its functional properties, ${ }^{[40]}$ as is also the possible oxygen loss in the crystalline structure. ${ }^{[19]}$

The peaks in the XRD diagram indicate a strong texture of the films in the out-of-plane direction. In the case of the cubic $\mathrm{SrVO}_{3}$, only 001 and 002 reflections are visible. For the $\mathrm{CaVO}_{3}$ thin films, the visible reflections can be identified as the $110_{0}$ and $220_{0}$ reflections, where o stands for orthorhombic. The $T_{\mathrm{G}}$ dependence of the out-of-plane lattice parameter $(c)$ is plotted in Figure 2c and summarized in Table S1, Supporting Information. When $T_{\mathrm{G}}$ increases, $c$ decreases continuously from 0.3811 to $0.3766 \mathrm{~nm}$ for CVO and from 0.3922 to $0.3857 \mathrm{~nm}$ for SVO. The observed values of $c_{\mathrm{pc}}$ are close to the bulk values, although slightly larger for the SVO films. The in-plane lattice parameter
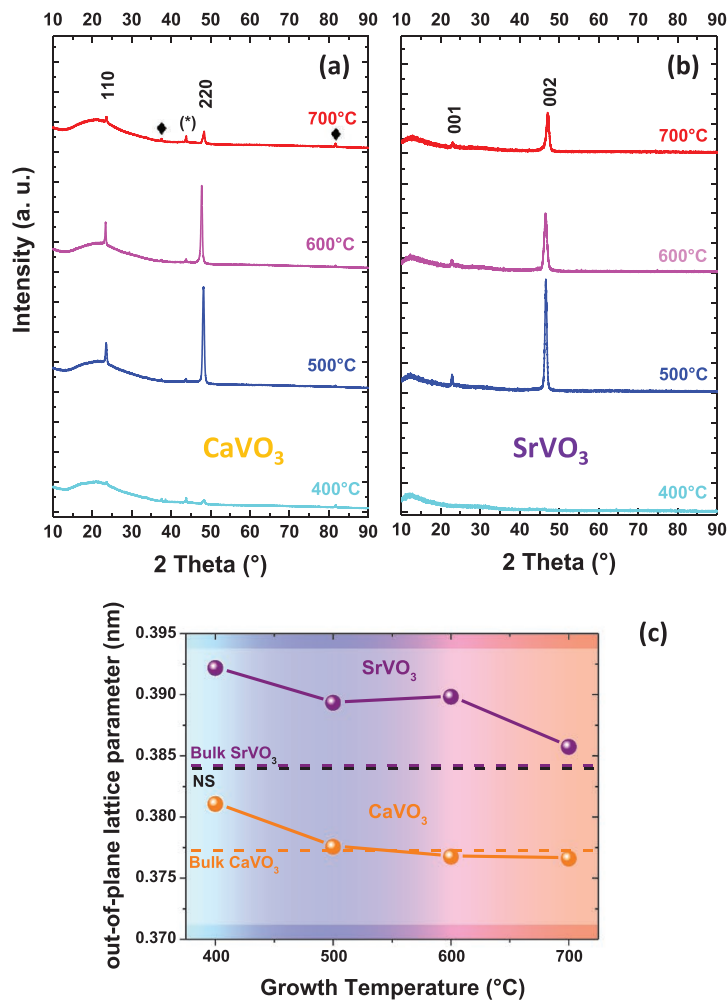

(c)

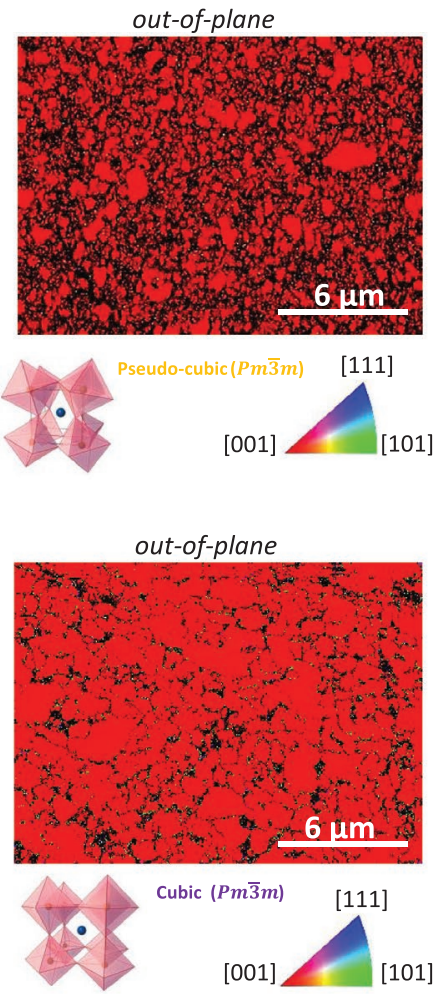

in-plane

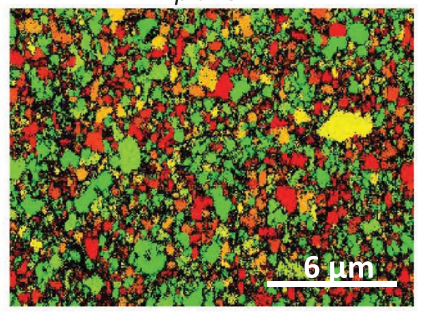

$\mathrm{CaVO}_{3} 500^{\circ} \mathrm{C}$

(d)

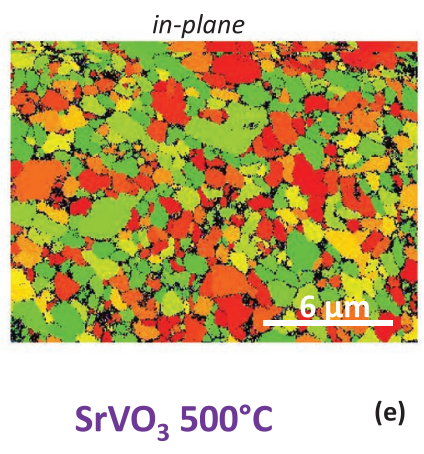

Figure 2. Structural and microstructural properties of textured vanadate thin films on glass. Specular $\theta-2 \theta \mathrm{X}$-ray diffractograms of CVO (a) and SVO (b) thin films grown on CNO NS/GS substrates from 400 up to $700{ }^{\circ} \mathrm{C}$. The symbols (*) and ( $)$ refer to parasite peaks stemming from the detector and the silver paste, respectively. c) Evolution of the out-of-plane lattice parameters as a function of the growth temperature. Out-of-plane and in-plane EBSD images of CVO (d) and SVO (e) thin films deposited at $500^{\circ} \mathrm{C}$ and their associated stereographic triangles. 
and consequently the cell volume also decrease with $T_{\mathrm{G}}$ (see Supporting Information). This observation is consistent with the cationic doping of the perovskite structure by out-diffusion of elements from the glass substrate and nanosheets evidenced by TEM for the highest temperatures.

The texture of the thin films induced by the NS is further investigated by electron backscatter diffraction (EBSD) mapping, for CVO (Figure 2d) and SVO (Figure 2e) grown at $T_{\mathrm{G}}=500{ }^{\circ} \mathrm{C}$. The mappings highlight the tessellated structure of the films similar to the SEM observations (see Supporting Information). Both CVO and SVO films are showing a preferential orientation along the [001] direction described in the pseudocubic system, confirming the XRD results. The in-plane EBSD maps show all directions between the [001] and [101] orientations, owing to the in-plane mosaic-like arrangement of NS, randomly dispersed on the surface. The black areas between the well-delimited nanosheets are related to non-diffracting domains, that is, amorphous films deposited on uncovered areas. From these EBSD micrographs, the proportion of crystallized phase $\left(P_{\mathrm{EBSD}}\right)$ (Figure $2 \mathrm{~d}$ and $2 \mathrm{e}$ ) was extracted and is equal to $65.0 \%$ (CVO) and $75.3 \%$ (SVO). These values match nicely with the coverage obtained previously. Thus, EBSD mapping emphasizes that the presence of NS is necessary to induce the crystallization of the highly textured vanadate films. This covering percentage of crystallized phase $(\approx 70 \%)$, is far higher than percolating thresholds in $2 \mathrm{D}$ conduction $(\approx 50 \%)$, which is an important condition for easy comparison of DC and optical conductivities.

The transport properties have been calculated from the optical measurements of the complex dielectric functions (DFs) $\varepsilon(\omega)=\varepsilon_{1}(\omega)+i \varepsilon_{2}(\omega)$ (as detailed in the Supporting Information), reported for $\mathrm{CVO}$ and $\mathrm{SVO}$ thin films grown at 400 and $500{ }^{\circ} \mathrm{C}$ in Figure 3a and 3b, respectively. The DFs of the films grown at $400{ }^{\circ} \mathrm{C}$ are typical of a near-amorphous film and will be taken as a reference for the amorphous phase. For the samples grown at a higher temperature, the DFs of the crystallized part is extracted by fitting concomitantly the proportion of the amorphous phase for each film. For films grown at $500{ }^{\circ} \mathrm{C}$ and higher, both DFs $\varepsilon_{1}$ and $\varepsilon_{2}$ are in good accordance with epitaxial vanadate thin films grown on single crystals ${ }^{[14,21]}$ or in their bulk form ${ }^{[16,46]}$ (see also the Supporting Information). In the near-infrared and infrared regions, we observe the onset of the absorption due to the free carriers, that is, the intraband transitions within the $t_{2 \mathrm{~g}}$ band. All these contributions confirm a good crystalline quality for the films grown at and above $500^{\circ} \mathrm{C}$.

The conduction properties of the films extracted from roomtemperature (RT) SE analyses and from DC transport measurements (Table S2, Supporting Information) are compared in Figure $3 \mathrm{c}-\mathrm{d}$. Vanadate films deposited at $400{ }^{\circ} \mathrm{C}$ are too resistive to be measured by DC transport, due to their poorly crystallized structure, ${ }^{[18]}$ which is confirmed by their high optical resistivity $\left(\rho_{\text {opt }}\right)$. For all well-crystallized films, the RT DC resistivity $\left(\rho_{\text {DC }}\right)$ is within a range from $3.65 \times 10^{-4}$ to $1.64 \times 10^{-3} \Omega \mathrm{cm}$ (Supporting Information). These values are consistent with those reported for bulk ${ }^{[15,47,48]}$ and thin films. ${ }^{[19]}$

Recalling that the amorphous phase is insulating, $\rho_{\mathrm{DC}}$ depends on the intrinsic DC resistivity $\rho_{D C}^{\text {cryst }}$ of the crystalline phase, its proportion $f=P_{\text {crystallized }} / 100$, and the quality of the geometrical connectivity between adjacent crystalline regions, and consequently on the geometry of conductivity paths between adjacent contacts. As we are clearly above the percolation threshold, effective medium theory applies (as detailed in
Dielectric functions

(a)

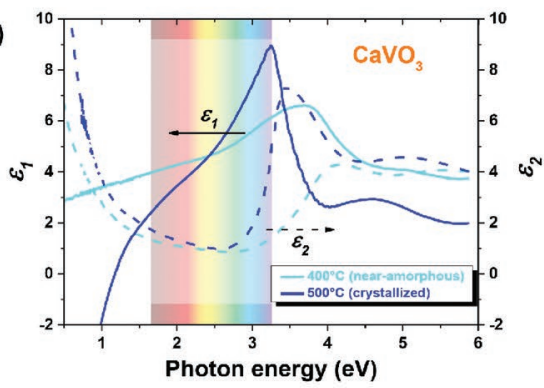

(b)

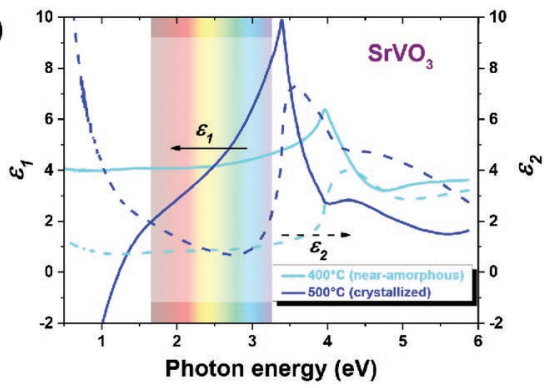

$D C$ and optical transport properties

(c)
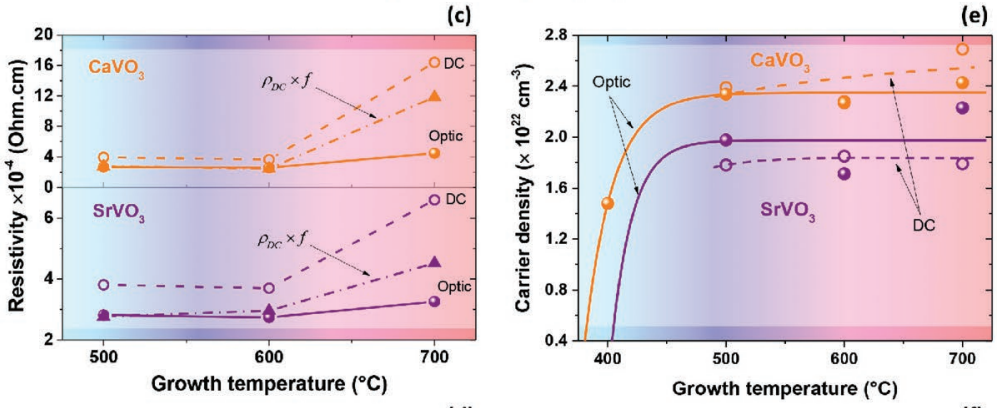

(d)

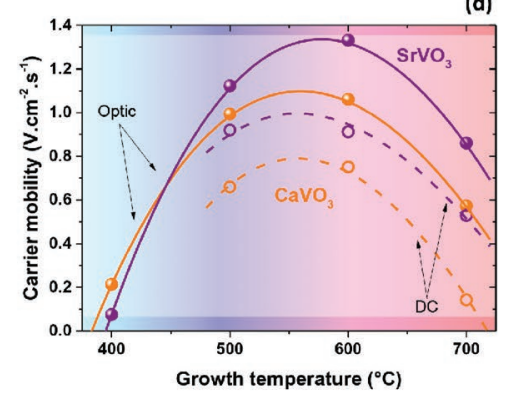

Figure 3. DC and optical transport properties of vanadate thin films on glass. Real part $\varepsilon_{1}$ and imaginary part $\varepsilon_{2}$ of the complex dielectric function of CVO (a) and SVO (b) thin films deposited on CNO NS/CS at $400^{\circ} \mathrm{C}$ (near-amorphous) and $500^{\circ} \mathrm{C}$ (crystallized) extracted from the SE measurements. All data in the $400-700{ }^{\circ} \mathrm{C}$ temperature range are reported. c-f): extracted values of SE measurements (solid lines) and Hall measurements (dashed lines) from CVO and SVO films deposited between 400 and $700{ }^{\circ} \mathrm{C}$ for c) the RT resistivity, d) the carrier density, e) the carrier mobility, and f) the effective mass (optical data only). For more information about the determination of $m * / m_{0}$, see Supporting Information. 
Supporting Information), which predicts that if the connectivity is maximal then $\rho_{D C}^{\text {cryst }}$ is simply the value of $\rho_{\mathrm{DC}} \times f$, because all crystalline parts are conduction paths. Moreover, DC measurements probe the long-range conductivity, while the optical measurements only probe the motion of the free electrons on a very short length scale (typically of a few nanometers at visible wavelengths). As a result, $\rho_{\text {opt }}$ corresponds to the resistivity within a single crystalline grain, while $\rho_{D C}^{\text {cryst }}$ also encompasses the inter-grain scattering, as well as the losses due to an imperfect geometrical connectivity between crystalline regions, so that in general $\rho_{D C}^{\text {cryst }} \geq \rho_{\text {opt }}$. For the samples grown at 500 and $600{ }^{\circ} \mathrm{C}, \rho_{D C}^{\text {cryst }}$ is very similar to $\rho_{\text {opt }}$ (Figure $3 \mathrm{C}$ ), allowing for two major conclusions: first, the connectivity of the crystalline network is nearly optimal (without "dead conduction paths" nor spatial segregation of the crystalline parts) and second, the scattering at the grain boundaries is negligible. For the sample grown at $700{ }^{\circ} \mathrm{C}, \rho_{\text {opt }}$ increases clearly compared to the 500 and $600{ }^{\circ} \mathrm{C}$ samples. The observed stronger intra-grain scattering can be explained by the poorer crystallinity evidenced by the XRD measurements and by the diffusion of cations from the substrate.

Considering the mobility as the most sensitive parameter reflecting the carrier scattering on defects, we compare in Figure $3 \mathrm{~d}$ the optical and DC carrier mobilities. The DC mobility $\left(\mu_{\mathrm{DC}}\right)$ is slightly lower than the extracted optical mobility $\left(\mu_{\text {opt }}\right)$, but both values remain very close and obey the same $T_{\mathrm{G}}$ dependence. Again, the maximum is observed in the 500-600 ${ }^{\circ} \mathrm{C}$ interval with $\mu_{\mathrm{DC}}=0.79(1.00) \mathrm{cm}^{2} \mathrm{~V}^{-1} \mathrm{~s}^{-1}$ and $\mu_{\mathrm{opt}}=$ $1.10(1.34) \mathrm{cm}^{2} \mathrm{~V}^{-1} \mathrm{~s}^{-1}$ for CVO (SVO) thin films is ascribed to the crystallinity enhancement just above the crystallization temperature and the degradation of the crystalline structure with even higher $T_{\mathrm{G}}$. Note that the SVO mobilities are somewhat higher than the CVO ones, which is consistent with the observations of Zhang et al. ${ }^{[14]}$ However, our values are about one order of magnitude lower than those measured on singlecrystalline thin films, ${ }^{[14,19]}$ due to the poly-crystalline character of our films.

The DC and optical carrier densities are presented in Figure 3e. The average values of $2.47 \times 10^{22} \mathrm{~cm}^{-3}\left(1.84 \times 10^{22} \mathrm{~cm}^{-3}\right)$ in DC measurements and $2.35 \times 10^{22} \mathrm{~cm}^{-3}\left(1.97 \times 10^{22} \mathrm{~cm}^{-3}\right)$ in optical measurements are reached for CVO (SVO) films. These values are very similar, and interestingly, the carrier density stays stable for $T_{\mathrm{G}}>500{ }^{\circ} \mathrm{C}$, indicating that the structural degradation of the films does not influence the carrier density. The observed values are slightly higher than the nominal density of $1.87 \times 10^{22} \mathrm{~cm}^{-3}\left(1.76 \times 10^{22} \mathrm{~cm}^{-3}\right)$ for one electron per unit cell of CVO (SVO), and at least one order of magnitude higher compared to semiconductor-based TCOs.

As the electronic correlations are of importance for the optical properties of vanadates, we determine the electron effective mass $m * / m_{0}$ by analyzing the SE data in the infrared range using the generalized Drude model (Supporting Information). As shown in Figure $3 \mathrm{f}, m^{*} / m_{0}$ of SVO films is between 2.61 and 3.34 and for CVO between 3.83 and 4.08. This enhancement reflects the stronger electronic correlations in $\mathrm{CVO}$, induced by the orthorhombic distortion. ${ }^{[49,50]}$ For the two curves, a maximum of $m^{*} / m_{0}$ is observed $\approx 600{ }^{\circ} \mathrm{C}$, as a trade-off between the enhancement of the crystalline quality for lower $T_{\mathrm{G}}$ and its deterioration for higher $T_{\mathrm{G}}$. Our values are in good agreement with bulk CVO and SVO (3.9 and 3.3, respectively), ${ }^{[16]}$ evidencing the relaxation of the strain thanks to the NS template in our films: indeed, the epitaxial strain has the effect of increasing the effective mass, varying from 4 to 5 , for SVO epitaxial thin films. ${ }^{[21]}$

Finally, from the DFs as a function of the frequency $\omega$, we also calculate the energy-loss function within the generalized Drude model (details given in Supporting Information). In this approach, assuming that the scattering rate and $m * / m_{0}$ are constant with $\omega$, the unscreened plasma frequency $\omega_{\mathrm{p}}$ is almost energy-independent. The screened plasma frequency $\omega_{\mathrm{p}} *=\omega_{\mathrm{p}} / \sqrt{ } \varepsilon_{\infty}$ can be extracted from the peak position of the energy-loss function (Figure $4 \mathrm{a}, \mathrm{b}$ ), but only the crystallized films exhibit a narrow plasmon peak. Near-amorphous CVO and SVO films $\left(T_{\mathrm{G}}=400^{\circ} \mathrm{C}\right)$ do not exhibit such a pronounced peak, as expected from their poorly conductive character. For crystallized films, $\omega_{\mathrm{p}}{ }^{*}=1.25(992 \mathrm{~nm})$ and $1.34 \mathrm{eV}(926 \mathrm{~nm})$ for $\mathrm{CVO}$ and SVO films grown at $500{ }^{\circ} \mathrm{C}$, respectively, pushing the absorption edge by the free carriers toward the IR region. It is also worth noting that $\omega_{\mathrm{p}}$ * decreases slightly with $T_{\mathrm{G}}$ increasing down to $1.20 \mathrm{eV}(1.30 \mathrm{eV})$ for $\mathrm{CVO}(\mathrm{SVO})$ at $700{ }^{\circ} \mathrm{C}$. In the case of strongly correlated systems, characterized by a spectral weight transfer from the Drude peak to higher energies, the shift of $\omega_{\mathrm{p}}{ }^{*}$ towards lower values indicates the destabilization of the strongly correlated charge carriers, in good agreement with the results from transport measurements described above. ${ }^{[16,46]}$

The pictures of the $40 \mathrm{~nm}$-thick CVO and SVO films deposited at different temperatures are shown in Figure 4c. All the samples are clearly transparent and exhibit the typical slightly green color due to the presence of the vanadate film, especially above $500{ }^{\circ} \mathrm{C}$. A darkening is observed at $700{ }^{\circ} \mathrm{C}$ both for SVO and CVO films. The transmission spectra of the films (Figure 4d,e) show very high transparency within the visible range, $\approx 70 \%$ to $\approx 75 \%$ beyond $450 \mathrm{~nm}$. Only the SVO film deposited at $700{ }^{\circ} \mathrm{C}$ shows lower values, confirmed by the darker grey color in Figure 4c. This darkening is consistent with the degradation of the structural and transport properties observed for high $T_{\mathrm{G}}$. Overall, our films have an excellent transmission, comparable to the ones of SVO and CVO single crystals ${ }^{[14,18,20]}$ or a-ITO films ${ }^{[3]}$ of the same thickness. To estimate the performances of the vanadate TCOs by considering both conducting and transparent behaviors, we calculate the Haacke figure of merit (FOM) defined as $\Phi_{\mathrm{TC}}=T^{10} / R_{\square}$, where $T$ is the transmission at $550 \mathrm{~nm}$ and $R_{\square}$ is the sheet resistance of the film ${ }^{[54]}$ (Table S3, Supporting Information). The FOM of CVO and SVO films are plotted as a function of $T_{\mathrm{G}}$ (Figure $4 \mathrm{f}$ ) and our values are compared to single crystal films ${ }^{[14,18]}$ and conventional TCOs. ${ }^{[3]}$ The $T_{\mathrm{G}}$ dependence of the FOM obeys a nearparabolic behavior with a maximum at $T_{\mathrm{G}} \approx 550{ }^{\circ} \mathrm{C}$ appearing as the optimal growth temperature for the integration of vanadate films on CNO NS. The highest FOM values $\Phi_{\mathrm{TC}}=2.5 \times$ $10^{-3} \Omega^{-1}\left(2.8 \times 10^{-3} \Omega^{-1}\right)$ for CVO (SVO) films are outreaching the values reported for epitaxial vanadate films deposited at $750-850{ }^{\circ} \mathrm{C}$ on single crystal $\left(\mathrm{LaAlO}_{3}\right)_{0.3}\left(\mathrm{Sr}_{2} \mathrm{TaAlO}_{6}\right)_{0.7}$ (LSAT) by molecular beam epitaxy $\left(\Phi_{\mathrm{TC}} \approx 1.0 \times 10^{-3} \Omega^{-1}\right),{ }^{[14]}$ but remain somewhat lower than epitaxial SVO thin films on LSAT grown by PLD $\left(\Phi_{\mathrm{TC}}=4.4 \times 10^{-3} \Omega^{-1}\right),{ }^{[18]}$ polycrystalline ITO $\left(\Phi_{\mathrm{TC}}=5.0\right.$ to $\left.5.8 \times 10^{-3} \Omega^{-1}\right),{ }^{[51,52]}$ and $\mathrm{AgNWs}\left(\Phi_{\mathrm{TC}}=1.7\right.$ to $\left.5.6 \times 10^{-3} \Omega^{-1}\right)^{[53]}$ films, for a similar thickness of $40 \mathrm{~nm}$. In the case of photovoltaic (PV) applications, the photons involved 

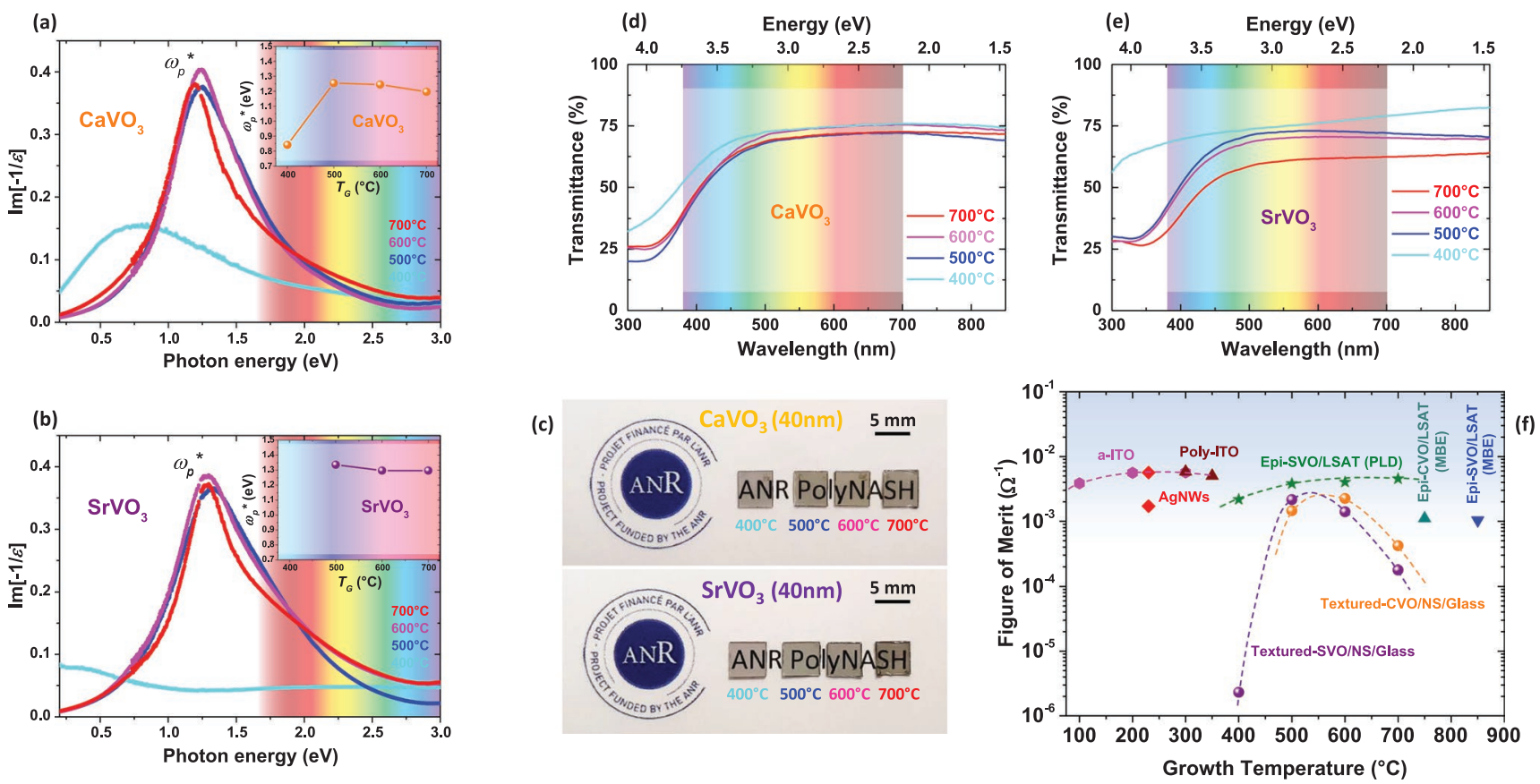

Figure 4. Optical properties of vanadate thin films on glass. Energy-loss functions $\operatorname{Im}[-1 / \varepsilon(\omega)]$ of CVO (a) and SVO (b) thin films obtained by the wavelength-by-wavelength fit in the framework of the generalized Drude model. The data around the photon energy of $1.3 \mathrm{eV}$ corresponds to the plasmon peak used to determine the screened plasma frequency plotted in the inset as a function of the growth temperature. c) Pictures of $40 \mathrm{~nm}$ thick CVO and SVO thin films deposited on CNO NS/GS between 400 and $700{ }^{\circ} \mathrm{C}$. Transmission spectra of CVO (d) and SVO thin films (e) deposited on CNO NS/GS at different $T_{\mathrm{C}}$. f) Haacke figure of merit of textured vanadate films on CNO NS/CS (orange and purple circles) and comparison with single crystals epitaxial SVO films (green stars ${ }^{[18]}$ and blue triangle ${ }^{[14]}$ ) and CVO films (cyan triangle ${ }^{[14]}$ ) grown onto adapted substrates, amorphous ITO (pink hexagons ${ }^{[3]}$ ), polycrystalline ITO (burgundy triangles ${ }^{[11,52]}$ ), and silver nanowires (red diamonds ${ }^{[3]}$ ) films of the same thickness.

in photoconversion are absorbed over a broad spectral range, including the yellow. The CVO and SVO coloration impact on potential PV application is estimated by the transmittance efficiency of the reference solar spectral global irradiance by the material thin film transmittance (see Supporting Information). The transmittance efficiency is correlated to a specific spectral irradiance over a framed spectral range taken between 300 and $997 \mathrm{~nm}$. In this range, ITO is the best TCO and transmits $84 \%$ of the global solar irradiance. The transmittance efficiency of $\mathrm{CVO}$ and SVO thin films grown at $500{ }^{\circ} \mathrm{C}$ are somewhat below with $66 \%$ and $68 \%$. These performances have to be compared to those of $\mathrm{SrNbO}_{3}$ (SNO) thin film, recently identified as a new performing TCO perovskite in ultraviolet range. ${ }^{[55,56]}$ For SNO, the transmittance efficiency reaches $73 \%$ and makes it a good candidate for PV applications. Overall, the FOM and transmittance efficiency of our vanadate films integrated on glass is in the same order of magnitude as standard TCOs, demonstrating their excellent perfomances and the sustainability of this new integrating process for the next generation of TCOs such as vanadates or SNO.

It should be pointed out here, that the NS approach for the vanadate growth on glass offers some unique optimization approaches. The glass substrate areas which are not covered by NS lead to amorphous regions in the vanadate film, with a higher optical transparency than the crystallized phase. The near-amorphous film deposited at $400{ }^{\circ} \mathrm{C}$ is $\approx 10 \%$ more transparent than the crystallized films in the near-UV and the nearIR parts of the visible spectrum (see Figure 4e). Therefore, the
FOM varying as $T^{10}$, it would be improved by the presence of small amounts of amorphous CVO or SVO regions, above the percolation threshold. The control of the NS coverage on the substrate can be easily gained through the parameters of the LB process. But even more, some devices, such as filters or anisotropic TCOs, require specific $2 \mathrm{D}$ geometries. This can be realized with the NS seed layer by photolithography patterning before film deposition to induce the selective crystallization of the film only in the NS covered areas. ${ }^{[30]}$ Therefore, the use of NS as growth templates offers new perspectives taking advantage of the enhanced transmission of amorphous areas and the possibility to induce an anisotropic electrical or optical conduction required for targeted applications.

\section{Conclusion and Perspectives}

Through the NS approach, the substrate preparation based on the LB process can be easily transferred to large-scale deposition techniques independently of the nature of the substrate. For example, LB roll-to-roll process, ${ }^{[57]}$ spray coating, ${ }^{[58]}$ inkjet printing, ${ }^{[59]}$ and drop casting ${ }^{[00]}$ large area deposition techniques have been successfully proved for the integration of NS on diverse substrates. Then, highly textured vanadates thin films can be grown on the prepared substrates by adapted thin deposition techniques, as large area solutions for $\mathrm{PLD}^{[6]}$ or radiofrequency sputtering in reductive conditions. ${ }^{[62]}$ The relatively low growth temperature of $500{ }^{\circ} \mathrm{C}$ is a crucial benefit for 
the integration of vanadates on thermally sensitive substrates, thereby facilitating a wide access to real devices. The induced texture by NS leads to exceptional transport and optical properties outreaching single crystal vanadate thin films and reaching the performances of conventional TCOs such as standard ITO, composite transparent conductors, or ultrathin metals. Our approach is particularly interesting for back electrode application where the electrode is in contact with the support. However, for top electrodes applications, the use of nanosheets could hinder the electrical contact between the active layer and electrode. Some development could be made by cationic doping of the nanosheets in order to decrease their intrinsic electrical resistivity and decrease the contact resistance. Moreover, we have built a SE model as a powerful tool able to characterize routinely the NS distribution over the surface and to extract the intrinsic transport and optical properties of integrated vanadate thin films. An important step towards the use of vanadate TCOs in devices is accomplished, contributing to the continued efforts to reduce the cost of devices due to the scarcity of indium and at the same time meeting the growing demand of TCOs in applications.

\section{Experimental Section}

The preparation of the exfoliated $\mathrm{Ca}_{2} \mathrm{Nb}_{3} \mathrm{O}_{10}{ }^{-}$nanosheets starting from powder of $\mathrm{KCa}_{2} \mathrm{Nb}_{3} \mathrm{O}_{10}$ oxide is described in detail in the references. ${ }^{[39,40]}$ The obtained nanosheets in colloidal solution were deposited on two side polished Eagle XG Corning glass substrates (strain point $669^{\circ} \mathrm{C}$ ) using the LB method with the help of a KSV NIMA instrument (scheme Figure 1b). The size of the glass substrates (hereafter named CS) was $10 \times 15 \mathrm{~mm}^{2}$, with a thickness of $0.5 \mathrm{~mm}$. After the dip coating, deposited NS/CS samples received a thermal treatment at $110^{\circ} \mathrm{C}$ during $90 \mathrm{~min}$ followed by an exposition to UV radiation during $45 \mathrm{~min}$ to enhance the adhesion of NS on GS and remove adsorbed residual solvents.

$\mathrm{CaVO}_{3}(\mathrm{CVO})$ and $\mathrm{SrVO}_{3}(\mathrm{SVO})$ thin films had been grown on cut $5 \times 5 \mathrm{~mm}^{2}$ NS/GS prepared samples by PLD. A KrF excimer laser $(\lambda=248 \mathrm{~nm})$ was used with a repetition rate of $3 \mathrm{~Hz}$ and a laser fluence adjusted at $1.6 \mathrm{~J} \mathrm{~cm}^{-2}$ to get a deposition rate close to $0.1 \AA$ per laser pulse. The laser beam was focused on a $\mathrm{Ca}_{2} \mathrm{~V}_{2} \mathrm{O}_{7}$ or a $\mathrm{Sr}_{2} \mathrm{~V}_{2} \mathrm{O}_{7}$ polycrystalline target prepared by a standard solid-state reaction. As the perovskite structure was not the most stable one in the $\mathrm{Ca}(\mathrm{Sr})-\mathrm{V}-\mathrm{O}$ system, ${ }^{[63,64]}$ the deposition was carried out under vacuum, that is, in reductive conditions, to reach the perovskite phase of the vanadate films. In order to investigate the influence of the temperature on the crystallization of the vanadate films, the substrate temperature $\left(T_{\mathrm{G}}\right)$ was varied between 400 and $700{ }^{\circ} \mathrm{C}$. The thickness of all deposited films was fixed to $40 \mathrm{~nm}$ by adjusting the deposition time. All thicknesses had been confirmed using X-ray reflectometry (XRR) (see Supporting Information). To avoid the oxidation of the deposited vanadate films, an ultrathin amorphous $\mathrm{LaAlO}_{3}$ (hereafter named a-LAO) capping layer was deposited at $150{ }^{\circ} \mathrm{C}$ by ablation of a $\mathrm{LaAlO}_{3}$ target under similar conditions. The thickness of this protective layer was set at $\approx 5 \mathrm{~nm}$ on the basis of a previous calibration.

The structural properties of the films were characterized by $\mathrm{X}$-ray diffraction (XRD) with a Philips X'Pert MRD diffractometer operating with monochromatic $\mathrm{Cu} \mathrm{K} \mathrm{K}_{\alpha 1}$ radiation $(\lambda=1.5406 \AA)$ and equipped with a Pixel detector. In-plane XRD was performed with a 5-circles SmartLab Rigaku with $\mathrm{Cu} \mathrm{K}_{\alpha 1,2}$ radiation $(\lambda=1.5418 \AA)$ and equipped with a HyPix-3000 detector. The morphology of the films was investigated by SEM with a Zeiss Supra 55 (FEG source) and AFM in tapping mode using a Pico SPM-LE microscope of Molecular Imaging and Bruker Dimension ICON 3100. EBSD mapping of sample surfaces were performed on the SEM microscope equipped with an ultra-sensitive high resolution detector from Bruker at an accelerating voltage of $15 \mathrm{kV}$. Data were treated by means of the Esprit software. The phase identification and orientations of CVO and SVO films were carried out based on bulk structural references in the computational method. The orthorhombic and cubic structure descriptions had been used for CVO and SVO films, respectively. TEM was performed by using a 2100 Jeol instrument operating at $200 \mathrm{kV}$ and equipped with a Gatan Orius 200D. Energy-dispersive X-ray spectroscopy (EDXS) was performed with an Oxford X-Max 80T silicon drift detector coupled to the TEM. The TEM samples were prepared by scratching their surface with a diamond tip. Small fragments of the sample containing film were then collected on a commercial TEM carbon-coated copper microgrid. The vanadates were particularly sensitive to the electron irradiation and all images had to be recorded in weak illumination conditions.

CVO samples grown at 500 and $700{ }^{\circ} \mathrm{C}$ had been characterized by XPS using time-resolved depth chemical profiling. These analyses were conducted on a Thermofisher Scientific Escalab $250 \times i$ equipped with a monochromated Al-K $\alpha$ anode $(1486.6 \mathrm{eV}$ ) and a dual flood gun (low energy electron and ion). High energy resolution spectral windows of interest were recorded with a $650 \mu \mathrm{m}$ spot size. The photoelectron detection was performed using a constant analyzer energy (CAE) mode ( $50 \mathrm{eV}$ pass energy) and a $0.1 \mathrm{eV}$ energy step. All the associated binding energies were corrected with respect to the Fermi level energy within the valence band spectral region assuming that CVO was a conductive oxide. XPS depth profiling required the use of the Thermofisher Scientific MACCIS DualBeam ion gun. Monoatomic Argon $\left(\mathrm{Ar}^{+}\right)$ions were accelerated to reach the sample with a kinetic energy of $4000 \mathrm{eV}$ and an angle of $30^{\circ}$ from the surface normal. The CVO thin films were irradiated on a square area of $1 \times 1 \mathrm{~mm}^{2}$. Quantification was performed based on the O1s, Cls, Ca2p and V2p3/2, La3d5, Nb3d and Si2s photopeak areas after a Shirley type background subtraction using the Thermofisher Scientific Avantage software and its "ALTHERMO1" library as sensitivity factor collection.

The temperature dependence of transport properties was determined by a four-probe method in the van der Pauw configuration from 5 to $370 \mathrm{~K}$ in a physical properties measurement system (PPMS) designed by Quantum Design. To determine the direct current (DC) carrier density and the DC mobility of carriers, Hall measurements were performed in the same temperature range by applying a magnetic field perpendicularly to the film plane within a range from -9 to $+9 \mathrm{~T}$. The optical properties were measured in the UV-visible-Near Infrared (UV-vis-NIR) range using a Lambda 1050 Perkin-Elmer spectrophotometer operating in reflection mode by means of the universal reflectance accessory (URA) and transmission mode between 200 and $1800 \mathrm{~nm}$. For all measurements, a pristine glass substrate was taken as reference.

All the films were characterized by variable angle spectroscopic ellipsometry (VASE) both in the UV-vis-NIR (from 0.7 to $5.9 \mathrm{eV}$ ) with a J. A. Woollam M2000XI, and in the IR range (from 0.04 to $0.7 \mathrm{eV}$ ) with a J. A. Woollam IR-Vase Mark II. The ellipsometric angles $\Psi$ and $\Delta$ were measured at an incident angle of $55^{\circ}, 65^{\circ}$, and $75^{\circ}$. The UV-vis-NIR and the IR data were then spliced together and analyzed concomitantly with the CompleteEase software supported by the J. A. Woolam Company. The ellipsometric measurements, the procedure, and the optical model developed in this work to take into account the different layers and their morphology are described in details in the Supporting Information.

\section{Supporting Information}

Supporting Information is available from the Wiley Online Library or from the author.

\section{Acknowledgements}

The authors gratefully acknowledge financial support of the French Agence Nationale de la Recherche (ANR) in the framework of the POLYNASH project (ANR-17-CE08-0012) and of the Normandie region 
and the European Funds FEDER through the project RIN RECHERCHE EVOL-PELIICAEN Contract No. 17P04263/17P04260 and CPER 2015-2020 MULTIMAT ScanMAT. The authors thank L. Gouleuf and J. Lecourt for the glass substrate preparation, S. Gascoin for her help for XRD characterizations, X. Larose for his assistance in SEM characterizations, $\mathrm{S}$. Froissart for her assistance for AFM measurements, and S. Duprey for his assistance for optical measurements.

\section{Conflict of Interest}

The authors declare no conflict of interest.

\section{Data Availability Statement}

Research data are not shared.

\section{Keywords}

correlated metals, nanosheets, perovskite thin films, spectroscopic ellipsometry, transparent conducting oxide

Received: August 12, 202 Published online:

[1] C. G. Granqvist, in Handbook of Transparent Conductors (Ed: D. S. Ginley), Springer US, Boston, MA 2011, pp. 353-423.

[2] R. A. Afre, N. Sharma, M. Sharon, M. Sharon, Rev. Adv. Mater. Sci. 2018, 53, 79.

[3] V. Teixeira, H. Cui, L. Meng, E. Fortunato, R. Martins, Thin Solid Films 2002, 420-421, 70.

[4] R. Kykyneshi, J. Zeng, D. P. Cann, in Handb. Transparent Conduct. (Ed: D.S. Ginley), Springer US, Boston, MA 2011, pp. 171-191.

[5] S. C. Dixon, D. O. Scanlon, C. J. Carmalt, I. P. Parkin, J. Mater. Chem. C 2016, 4, 6946.

[6] T. E. Graedel, E. M. Harper, N. T. Nassar, P. Nuss, B. K. Reck, Proc. Natl. Acad. Sci. U. S. A. 2015, 112, 4257.

[7] C. H. Choi, J. Cao, F. Zhao, Resour., Conserv. Recycl. 2016, 114, 59.

[8] T. Watari, K. Nansai, K. Nakajima, Resour., Conserv. Recycl. 2020, $155,104669$.

[9] K. Ellmer, J. Phys. D: Appl. Phys. 2000, 33, R17.

[10] A. J. Freeman, K. R. Poeppelmeier, T. O. Mason, R. P. H. Chang, T. J. Marks, MRS Bull. 2000, 25, 45.

[11] T. Minami, Semicond. Sci. Technol. 2005, 20, S35.

[12] E. Fortunato, D. Ginley, H. Hosono, D. C. Paine, MRS Bull. 2007, 32, 242.

[13] C. G. Granqvist, Sol. Energy Mater. Sol. Cells 2007, 91, 1529.

[14] L. Zhang, Y. Zhou, L. Guo, W. Zhao, A. Barnes, H.-T. Zhang, C. Eaton, Y. Zheng, M. Brahlek, H. F. Haneef, N. J. Podraza, M. H. W. Chan, V. Gopalan, K. M. Rabe, R. Engel-Herbert, Nat. Mater. 2015, 15, 204.

[15] P. Dougier, J. C. C. Fan, J. B. Goodenough, J. Solid State Chem. 1975, 14, 247.

[16] H. Makino, I. H. Inoue, M. J. Rozenberg, Y. Aiura, I. Hase, S. Onari, arXiv:cond-mat/9801086 1998.

[17] J. A. Moyer, C. Eaton, R. Engel-Herbert, Adv. Mater. 2013, 25, 3578.

[18] A. Boileau, A. Cheikh, A. Fouchet, A. David, R. Escobar-Galindo, C. Labbé, P. Marie, F. Gourbilleau, U. Lüders, Appl. Phys. Lett. 2018, 112, 021905
[19] A. Boileau, A. Cheikh, A. Fouchet, A. David, C. Labbé, P. Marie, F. Gourbilleau, U. Lüders, Adv. Opt. Mater. 2019, 7, 1801516.

[20] M. Mirjolet, F. Sánchez, J. Fontcuberta, Adv. Funct. Mater. 2019, 29, 1808432.

[21] M. Mirjolet, H. B. Vasili, L. I. López-Conesa, S. Estradé, F. Peiró, J. Santiso, F. Sánchez, P. Machado, P. Gargiani, M. Valvidares, J. Fontcuberta, Adv. Funct. Mater. 2019, 29, 1904238.

[22] L. Shoham, M. Baskin, M. Han, Y. Zhu, L. Kornblum, Adv. Electron. Mater. 2020, 6, 1900584.

[23] P. Zhang, I. Wyman, J. Hu, S. Lin, Z. Zhong, Y. Tu, Z. Huang, Y. Wei, Mater. Sci. Eng.: B 2017, 223, 1.

[24] D. L. Ritums, N. J. Wu, X. Chen, D. Liu, A. Ignatiev, AIP Conf. Proc. 1998, 420, 672.

[25] P. W. Yip, K. H. Wong, MRS Proc. 2000, 623, 347.

[26] H. Ishiwara, K. Jyokyu, Jpn. J. Appl. Phys. 1991, 30, L2059.

[27] H. Nagata, Thin Solid Films 1993, 224, 1.

[28] B. K. Moon, H. Ishiwara, Appl. Phys. Lett. 1995, 67, 1996.

[29] B. K. Moon, E. Tokumitsu, H. Ishiwara, Mater. Sci. Eng.: B 1996, 41, 157

[30] M. Nijland, S. Thomas, M. A. Smithers, N. Banerjee, D. H. A. Blank, G. Rijnders, J. Xia, G. Koster, J. E. ten Elshof, Adv. Funct. Mater. 2015, 25, 5140 .

[31] B.-W. Li, M. Osada, Y. Ebina, K. Akatsuka, K. Fukuda, T. Sasaki, ACS Nano 2014, 8, 5449.

[32] H. Yuan, R. Lubbers, R. Besselink, M. Nijland, J. E. ten Elshof, ACS Appl. Mater. Interfaces 2014, 6, 8567.

[33] T. Shibata, Y. Ebina, T. Ohnishi, K. Takada, T. Kogure, T. Sasaki, Cryst. Growth Des. 2010, 10, 3787.

[34] J. Kimura, I. Takuwa, M. Matsushima, T. Shimizu, H. Uchida, T. Kiguchi, T. Shiraishi, T. J. Konno, T. Shibata, M. Osada, T. Sasaki, H. Funakubo, Sci. Rep. 2016, 6, 20713.

[35] S.-H. Kweon, M. Im, W.-H. Lee, S. Nahm, J.-W. Choi, S.-J. Hwang, J. Mater. Chem. C 2016, 4, 178.

[36] T. Shibata, H. Takano, Y. Ebina, D. S. Kim, T. C. Ozawa, K. Akatsuka, T. Ohnishi, K. Takada, T. Kogure, T. Sasaki, J. Mater. Chem. C 2014, 2, 441.

[37] M. Nijland, S. Kumar, R. Lubbers, D. H. A. Blank, G. Rijnders, G. Koster, J. E. ten Elshof, ACS Appl. Mater. Interfaces 2014, 6, 2777.

[38] V. Bouquet, F. Baudouin, V. Demange, S. Députier, S. Ollivier, L. Joanny, L. Rault, A. Fouchet, M. Guilloux-Viry, Thin Solid Films 2020, 693, 137687.

[39] A. Boileau, M. Dallocchio, F. Baudouin, A. David, U. Lüders, B. Mercey, A. Pautrat, V. Demange, M. Guilloux-Viry, W. Prellier, A. Fouchet, ACS Appl. Mater. Interfaces 2019, 11, 37302.

[40] F. Baudouin, V. Demange, S. Ollivier, L. Rault, A. S. Brito, A. S. Maia, F. Gouttefangeas, V. Bouquet, S. Députier, B. Bérini, A. Fouchet, M. Guilloux-Viry, Thin Solid Films 2020, 693, 137682.

[41] J. J. Manguele, F. Baudouin, C. Cibert, B. Domengès, V. Demange, M. Guilloux-Viry, A. Fouchet, G. Poullain, SN Appl. Sci. 2020, 2 , 453.

[42] M. J. Rey, P. Dehaudt, J. C. Joubert, B. Lambert-Andron, M. Cyrot, F. Cyrot-Lackmann, J. Solid State Chem. 1990, 86, 101.

[43] H. Falcón, J. A. Alonso, M. T. Casais, M. J. Martínez-Lope, J. Sánchez-Benítez, J. Solid State Chem. 2004, 177, 3099.

[44] J. Hart, Innovation in Global Industries, National Research Council, Washington, DC 2008.

[45] C. V. Cushman, B. A. Sturgell, A. C. Martin, B. M. Lunt, N. J. Smith, M. R. Linford, Surf. Sci. Spectra 2016, 23, 55.

[46] R. J. O. Mossanek, M. Abbate, P. T. Fonseca, A. Fujimori, H. Eisaki, S. Uchida, Y. Tokura, Phys. Rev. B 2009, 80, 195107.

[47] Y. C. Lan, X. L. Chen, M. He, J. Alloys Compd. 2003, 354, 95.

[48] T. Maekawa, K. Kurosaki, S. Yamanaka, J. Alloys Compd. 2006, 426, 46.

[49] T. Yoshida, M. Hashimoto, T. Takizawa, A. Fujimori, M. Kubota, K. Ono, H. Eisaki, Phys. Rev. B 2010, 82, 085119. 
[50] S. Backes, T. C. Rödel, F. Fortuna, E. Frantzeskakis, P. Le Fèvre, F. Bertran, M. Kobayashi, R. Yukawa, T. Mitsuhashi, M. Kitamura, K. Horiba, H. Kumigashira, R. Saint-Martin, A. Fouchet, B. Berini, Y. Dumont, A. J. Kim, F. Lechermann, H. O. Jeschke, M. J. Rozenberg, R. Valentí, A. F. Santander-Syro, Phys. Rev. B 2016, 94, $241110(\mathrm{R})$.

[51] H. Kim, J. S. Horwitz, G. Kushto, A. Piqué, Z. H. Kafafi, C. M. Gilmore, D. B. Chrisey, J. Appl. Phys. 2000, 88, 6021.

[52] H.-C. Lee, J.-Y. Seo, Y.-W. Choi, D.-W. Lee, Vacuum 2003, 72, 269.

[53] B. T. Camic, H. I. Jeong, M. H. Aslan, A. Kosemen, S. Kim, H. Choi, F. Basarir, B. R. Lee, Nanomaterials 2019, 10, 46.

[54] G. Haacke, J. Appl. Phys. 1976, 47, 4086.

[55] Y. Park, J. Roth, D. Oka, Y. Hirose, T. Hasegawa, A. Paul, A. Pogrebnyakov, V. Gopalan, T. Birol, R. Engel-Herbert, Commun. Phys. 2020, 3, 102.
[56] J. Roth, A. Paul, N. Goldner, A. Pogrebnyakov, K. Agueda, T. Birol, N. Alem, R. Engel-Herbert, ACS Appl. Mater. Interfaces 2020, 12, 30520.

[57] X. Li, J. F. Gilchrist, Langmuir 2016, 32, 1220.

[58] P. Li, T.-C. Huang, K. L. White, S. Hawkins, M. Kotaki, R. Nishimura, H.-J. Sue, RSC Adv. 2015, 5, 102633.

[59] S. Chung, K. Cho, T. Lee, Adv. Sci. 2019, 6, 1801445.

[60] Y. Shi, M. Osada, Y. Ebina, T. Sasaki, ACS Nano 2020, 14, 15216.

[61] J. A. Greer, J. Phys. D: Appl. Phys. 2014, 47, 034005.

[62] D. H. Jung, H. S. So, H. Lee, J. Vac. Sci. Technol. A 2019, 37, 021507.

[63] J. Macías, A. A. Yaremchenko, J. R. Frade, J. Alloys Compd. 2014, 601, 186.

[64] B. Bérini, V. Demange, M. Bouttemy, E. Popova, N. Keller, Y. Dumont, A. Fouchet, Adv. Mater. Interfaces 2016, 3, 1600274. 